\title{
Proceso de identificación de stakeholders de programas de educación financiera en Ecuador
}

\section{Stakeholder identification process of financial education programs in Ecuador}

Miguel Cueva Estrada

Jorge Montecel Shiguango

Universidad de Guayaquil, Ecuador

Michelle Viera Romero

Universidad Potsdam, Alemania

Universidad de Guayaquil, Ecuador

Autor para correspondencia: nathaelcueva@gmail.com, jorge.montecelsh@ug.edu.ec, michelle.vierarom@ug.edu.ec

Fecha de recepción: 14 de Junio de 2017 - Fecha de aceptación: 15 de Noviembre de 2017

Resumen: Luego de la dolarización, la economía ecuatoriana ha gozado de una relativa estabilidad demostrando un crecimiento sostenido que ha tenido como resultado un incremento de su clase media. Este crecimiento económico trae consigo la necesidad de que las personas sepan cómo manejar sus finanzas personales y beneficiarse de los mercados financieros más desarrollados. En este sentido, las iniciativas de educación financiera, pueden convertirse en un complemento importante de los procesos de inclusión y demás medidas para la reducción de la pobreza. En consecuencia, el Estado ecuatoriano ha dispuesto la implementación de programas de educación financiera orientados a contar con una ciudadanía mejor informada. De parte de las entidades financieras que han puesto en marcha estos programas, se puede identificar un beneficio adicional que corresponde a cubrir la responsabilidad social que estas instituciones tienen con la comunidad derivado de las actividades que realizan. Una revisión a distintos documentos normativos, consultas a expertos y una evaluación del grado de poder, urgencia y legitimidad que representan las partes interesadas en lo referente a la educación financiera en el Ecuador, ha permitido identificar a los principales stakeholders quienes se convertirán en los líderes de la cooperación interinstitucional para la amplia difusión e implementación de estos programas entre los grupos objetivos.

Palabras claves: educación financiera; stakeholders; responsabilidad social; gobernanza; ecuador Abstract: After dollarization, the Ecuadorian economy has enjoyed relative stability demonstrating sustained growth that has resulted in an increase in its middle class. This economic growth brings with it the need for people to know how to manage their personal finances and benefit from more developed financial markets. In this sense, financial education initiatives can become an important complement to inclusion processes and other measures to reduce poverty. Consequently, the Ecuadorian State has arranged the implementation of financial education programs aimed at having a better informed citizenship. On the part of the financial entities that have implemented these programs, an additional benefit can be identified that corresponds to cover 
the social responsibility that these institutions have with the community derived from the activities they carry out. A review of different normative documents, consultations with experts and an assessment of the degree of power, urgency and legitimacy that stakeholders represent in relation to financial education in Ecuador, has allowed identifying the main stakeholders who will become the leaders of inter-institutional cooperation for the wide dissemination and implementation of these programs among the target groups.

Key words: financial education; stakeholders; social responsibility; governance; ecuador

\section{Introducción}

Para la población ecuatoriana es bastante delicado discutir respecto de temas financieros, sobre por la desconfianza que generó la crisis bancaria de finales de los $90 \mathrm{~s}$, el posterior cambio de moneda donde se asumió el dólar y las continuas modificaciones a las que es sometido su marco general regulatorio.

En el Ecuador desde hace pocos años se ha venido trabajando con la implementación y promoción de lo que se conoce como Educación Financiera. Este lineamiento ha sido incluido en el Plan Nacional del Buen Vivir 2013-2017, es decir el máximo instrumento de planificación del país, a partir del cual se elaboran los distintos programas y proyectos que permitan alcanzar los objetivos propuestos en este plan de desarrollo.

En este proceso de generación de políticas públicas, la literatura especializada identifica de manera clara las siguientes etapas: surgimiento del problema e inclusión en la agenda, diseño del programa, implementación y evaluación de la política (Weimar \& Vining, 1989).

Cada uno de estas etapas está de manera permanente influenciada por la intervención de los distintos actores sociales, públicos y privados, llamados también stakeholders, partes interesadas o grupos de interés, y son quienes tienen distintas expectativas, intereses, obligaciones, deberes y derechos con respecto a la directriz que se pretende implementar. (Como se cita en Falçao Martins e Fontes Filho, 1999).

Es por ese motivo que el presente documento pretende primero identificar a los stakeholders y estudiar el grado de interés respecto de lo que corresponde a la implementación de programas de Educación Financiera como parte de una política de ciudadanía responsable y debidamente informada, lo que da cumplimiento a la responsabilidad social asumida por las entidades financieras.

\section{La Educación Financiera en el Ecuador}

Como concepto, la Educación Financiera ha ido ganando terreno en los últimos años por cuanto dicha información es necesaria para el público con la finalidad que conozca cómo acceder al crédito y cómo utilizar estos recursos correctamente (García, N. Grifoni, A. \& López, J., 2013).

En la actualidad, los productos financieros que se le presentan a los usuarios son cada vez más diversos, más complejos e implican un mayor riesgo al involucrar nuevas tecnologías como son tarjetas, celulares, banca electrónica, etc. (Coates, 2009). Por este motivo, es de interés del 
Estado contar con "ciudadanos instruidos e informados que tomen decisiones económicas y financieras sensatas (...) que contribuyan a la estabilidad macroeconómica" (Coates, 2009) del país.

Además, se hace necesario reforzar algunos conocimientos y habilidades y acompañar con la orientación necesaria respecto de los riesgos asociados con cada decisión financiera de los individuos, ya que muchas veces se trata de decisiones de vida (educación, vivienda, seguridad social, planes de salud, etc.).

El Gobierno del Ecuador, en un primer momento hace referencia a la educación financiera en el Plan Nacional del Buen Vivir 2013-2017, Objetivo 4.9 literal m) "Generar programas y contenidos educativos que difundan y fortalezcan la educación financiera". Para que más adelante, sea la Junta Bancaria quien resuelve a través de la resolución No. JB-2013-2393 del 22 de enero de 2013 disponer que las entidades financieras controladas por la Superintendencia de Bancos y Seguros desarrollen Programas de Educación Financiera (PEF) en beneficio de sus clientes y público en general.

El objetivo final de esta iniciativa es dotar a la población de conocimiento que apoyen la toma de decisiones sobre sus finanzas personales y familiares; seguros y seguridad social, que amplíen sus oportunidades de participación informada y responsable a lo largo de la vida económica de las personas.

Adicional a estos documentos normativos que rigen el quehacer institucional del país, está la Ley de Defensa del Consumidor junto con la Resolución No. SB-2015-665 como documentos que reglamentan los programas de educación financiera dentro del país.

Lamentablemente, ninguno de los instrumentos mencionados define con claridad quién es el principal regulador de la implementación de esta práctica. En todo caso, lo que se puede inferir es que debido al ámbito de acción, el compromiso ha recaído en principio dentro de la Junta Bancaria quien distribuye responsabilidades entre actores tan diversos como el Ministerio de Educación, la Superintendencia de Bancos, la Defensoría del Pueblo, etc. Más adelante, será la Superintendencia de Bancos quien asumirá este liderazgo delineando algunas directrices para el diseño de los programas.

Es así que al no existir un instrumento que delimite quién debe asumir la formulación, implementación y evaluación de los programas de educación financiera, eso se convierte en el punto de partida de este artículo y así buscar el beneficio de la sociedad con una amplia promoción de estas prácticas apoyado en sus partes interesadas, quienes también pueden incorporar estas prácticas a su portafolio de proyectos o actividades que forman parte de sus programas de responsabilidad social empresarial.

Por todo lo anteriormente citado es importante para los programas de educación financiera en el país, tener claramente identificados sus stakeholders, entendiendo lo importante que resulta para la correcta implementación de la política el comprender los intereses de estas partes y el incentivo que representa para ellos desde el punto de vista de la responsabilidad social. Esto le dará mayor legitimidad y sostenibilidad en el tiempo ya que podrá conocer a los 
stakeholders, comprenderlos, involucrarlos en diálogos, entender sus necesidades, comprender sus demandas y requerimientos, identificar las expectativas que han desarrollado, propendiendo a la transparencia mediante el hábito de la rendición de cuentas.

\section{Metodología}

El presente trabajo de investigación es de carácter exploratorio, dado que tiene por objeto conocer las características de los stakeholders involucrados en la implementación de los programas de educación financiera, así como de la naturaleza y tipo de interacciones que se presentan entre ellos. Es decir, que la unidad de análisis son los Programas de Educación Financiera vigentes.

Para este propósito, se utilizan fuentes de carácter secundario, páginas web de distintas entidades que describan a los stakeholders y los programas de educación financiera, resoluciones e informes que son de dominio público y que proporcionen información relevante a la investigación.

Adicionalmente, para la categorización de los stakeholders, se realizarán entrevistas a un grupo de expertos en el sistema financiero ecuatoriano en base a su experiencia profesional y su independencia. Toda esta información será luego procesada de acuerdo con el Modelo de Mitchell, Agle y Wood (1997) para la identificación de los distintos stakeholders según los atributos de poder, legitimidad y urgencia.

\section{El Modelo de Mitchell, Agle y Wood}

Este procedimiento tiene como finalidad identificar a los distintos stakeholders a través de la operacionalización de tres atributos básicos que son:

1. El poder suficiente para influir en la organización.

2. La legitimidad para poder mantener una relación entre ellos y la organización.

3. La urgencia de la pretensión que tengan estos grupos de interés (Mitchell, Agle, \& Wood, 1997).

Según lo que cada stakeholder representa en función de la naturaleza o de la posición relativa de estos actores, se les asignará un valor asociado con su preponderancia o importancia de acuerdo a los comentarios expresados por el panel de expertos encuestados respecto del poder que pudieran ejercer, su legitimidad y la urgencia.

Ya que según Mitchell et al (1997), si se combinan estos tres atributos, surgen diferentes grupos de partes interesadas, con diferentes expectativas respecto al objeto de interés, lo que se convierte en un instrumento de apoyo para la toma de decisiones por parte de los líderes de la iniciativa analizada. Ver Gráfico $\mathrm{N}^{\circ} 1$. 


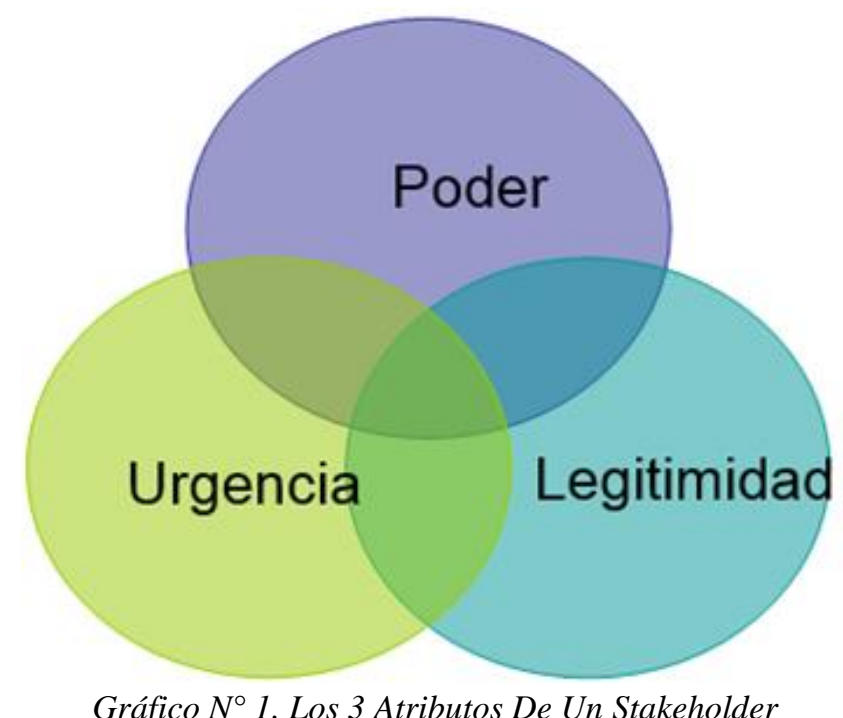

Fuente: Adaptado de Falçao Martins e Fontes Filho, 1999

\section{Identificación de Stakeholders - Resultados}

Los programas de educación financiera son responsabilidad de múltiples actores. De manera general se puede pensar en bancos, autoridades reguladoras del sistema financiero, y la ciudadanía en general, pero lo cierto es que involucra los esfuerzos de muchos más como son el Ministerio de Educación, la Defensoría del Pueblo, etc. Por este motivo, se hace necesario identificar a los principales involucrados, los más visibles y cercanos a los temas, y que serían los que pueden intervenir de manera directa en la implementación de estos programas que a fin de cuentas van a convertirse en una política del Estado en beneficio de sus ciudadanos.

Tomando en cuenta la fundamentación conceptual propuesta en el trabajo de Mitchell, Agle \& Wood (1997), se procede a clasificar la importancia de los actores midiendo y valorando la existencia de los atributos de poder, legitimidad y urgencia, determinado para ello el índice de preponderancia.

Donde el poder es medido según la disponibilidad de este recurso y la influencia a ejercer, por lo que se asigna el valor de 1 no existe ninguna sensibilidad ante el recurso descrito, 2 cuando la sensibilidad es media, y 3 cuando la sensibilidad es extrema.

De manera similar, la legitimidad se estima con un valor de 0 cuando las acciones de los actores son indeseables, 1 cuando las acciones del actor son mínimamente deseables por la sociedad, 2 cuando son medianamente deseables y 3 cuando son altamente deseables y legítimas.

Por último, la urgencia es estimada con un valor de 0 cuando existe una total aceptación de la demora y una baja sensibilidad a lo crítico de la actividad, 1 cuando la aceptación y la sensibilidad están por debajo del promedio, 2 cuando la sensibilidad está por encima del promedio, y 3 cuando existe un total rechazo a la demora. 
Preliminarmente, una vez que se revisa la documentación normativa se identifica a un primer grupo de 27 stakeholders desglosados desde los distintos documentos que orientan la implementación de la Educación Financiera en el país, como son el mismo Plan Nacional del Buen Vivir 2013 - 2017, la Ley Orgánica de Defensa del Consumidor, las resoluciones de la Junta Bancaria y de la Superintendencia de Bancos.

Luego de lo cual se presenta dicha información a los expertos, para que con su competencia técnica e independencia puedan determinar cuáles son las partes interesadas más relevantes. Finalmente, logran agrupar cada una de las partes interesadas en 13 categorías que se detallan a continuación:

1. Superintendencia de Bancos

2. Directorio o Consejo de Administración

3. Junta de Accionistas o Socios

4. Gerencia de la Institución Financiera Privada o Pública

5. Unidad responsable de los programas de Educación Financiera en la organización

6. Colaboradores de la Institución Financiera pública, privada o de seguridad social

7. Cliente de la Institución Financiera o de seguridad social

8. Público Objetivo Niños Preescolares (5-8 años)

9. Público Objetivo Preadolescentes (9-12 años)

10. Público Objetivo Jóvenes (13-17 años)

11. Público Objetivo Jóvenes Adultos (18-24 años)

12. Público Objetivo Adultos (25-64 años)

13. Público Objetivo Adultos Mayores (más de 65 años)

Para efectos prácticos cada uno de ellos recibe una abreviatura, se le asignan roles de acuerdo a sus actividades. Así tenemos que algunos cumplen un rol político como la Superintendencia de Bancos. Otros son clientes, observadores, supervisores, etc.

Poco a poco, de acuerdo a lo descrito por los expertos se asignan los grados de importancia que representa cada uno de los atributos de urgencia, legitimidad y poder con respecto a cada uno de los 13 stakeholders definidos. Dicha puntuación se ve reflejada en la Tabla $\mathrm{N}^{\circ} 1$ y en el respectivo Gráfico $\mathrm{N}^{\circ} 2$ que aparecen a continuación: 
Tabla $\mathbf{N}^{\circ}$ 1. Matriz De Consolidación

\begin{tabular}{|c|c|c|c|c|c|}
\hline Clave & $\begin{array}{l}\text { Categoría de } \\
\text { Stakeholder }\end{array}$ & $\begin{array}{c}\text { Grado } \\
\text { de Poder }\end{array}$ & $\begin{array}{c}\text { Grado de } \\
\text { Legitimidad }\end{array}$ & $\begin{array}{l}\text { Grado de } \\
\text { Urgencia }\end{array}$ & $\begin{array}{c}\text { Indice de } \\
\text { Preponderancia }\end{array}$ \\
\hline $\begin{array}{l}\text { Superintendencia de } \\
\text { Bancos }\end{array}$ & Político & 1.93 & 1.36 & 1.31 & 2.53 \\
\hline $\begin{array}{l}\text { Directorio o Consejo de } \\
\text { Administración }\end{array}$ & Fondeador & 0.32 & 0.91 & 0.88 & 0.19 \\
\hline $\begin{array}{l}\text { Junta de Accionistas o } \\
\text { Socios }\end{array}$ & Cliente & 0.64 & 0.45 & 0.29 & 0.06 \\
\hline $\begin{array}{l}\text { Gerencia de la IFI privada } \\
\text { o pública }\end{array}$ & Cliente & 0.64 & 0.45 & 0.58 & 0.12 \\
\hline $\begin{array}{l}\text { Unidad responsable de los } \\
\text { programas de EF en la } \\
\text { organización }\end{array}$ & Cliente & 0.32 & 1.36 & 0.58 & 0.19 \\
\hline $\begin{array}{l}\text { Colaboradores de la IFI } \\
\text { pública, privada o de } \\
\text { seguridad social }\end{array}$ & Observadores & 0.32 & 0.15 & 0.15 & 0.01 \\
\hline $\begin{array}{l}\text { Cliente de IFI o de } \\
\text { seguriddad social }\end{array}$ & Estratégico & 1.44 & 0.15 & 1.31 & 0.21 \\
\hline $\begin{array}{l}\text { Público Objetivo Niños } \\
\text { Preescolares (5-8) }\end{array}$ & Operativo & 1.12 & 1.36 & 1.31 & 1.48 \\
\hline $\begin{array}{l}\text { Público Objetivo } \\
\text { Preadolescentes }(9-12)\end{array}$ & Administrativo & 1.28 & 1.36 & 1.31 & 1.69 \\
\hline $\begin{array}{l}\text { Públio objetivo Jóvenes } \\
(13-17)\end{array}$ & Administrativo & 1.28 & 1.36 & 1.31 & 1.69 \\
\hline $\begin{array}{l}\text { Público Objetivo Jovenes } \\
\text { Adultos ( } 18-24)\end{array}$ & Estratégico & 1.28 & 1.36 & 1.31 & 1.69 \\
\hline $\begin{array}{l}\text { Público Objetivo Adultos } \\
\text { (25-64) }\end{array}$ & Estratégico & 1.12 & 1.36 & 1.31 & 1.48 \\
\hline $\begin{array}{l}\text { Público Objetivo Adultos } \\
\text { Mayores de }(65 \text { y }+)\end{array}$ & Supervisión & 1.28 & 1.36 & 1.31 & 1.69 \\
\hline
\end{tabular}

Fuente: Elaboración Propia 


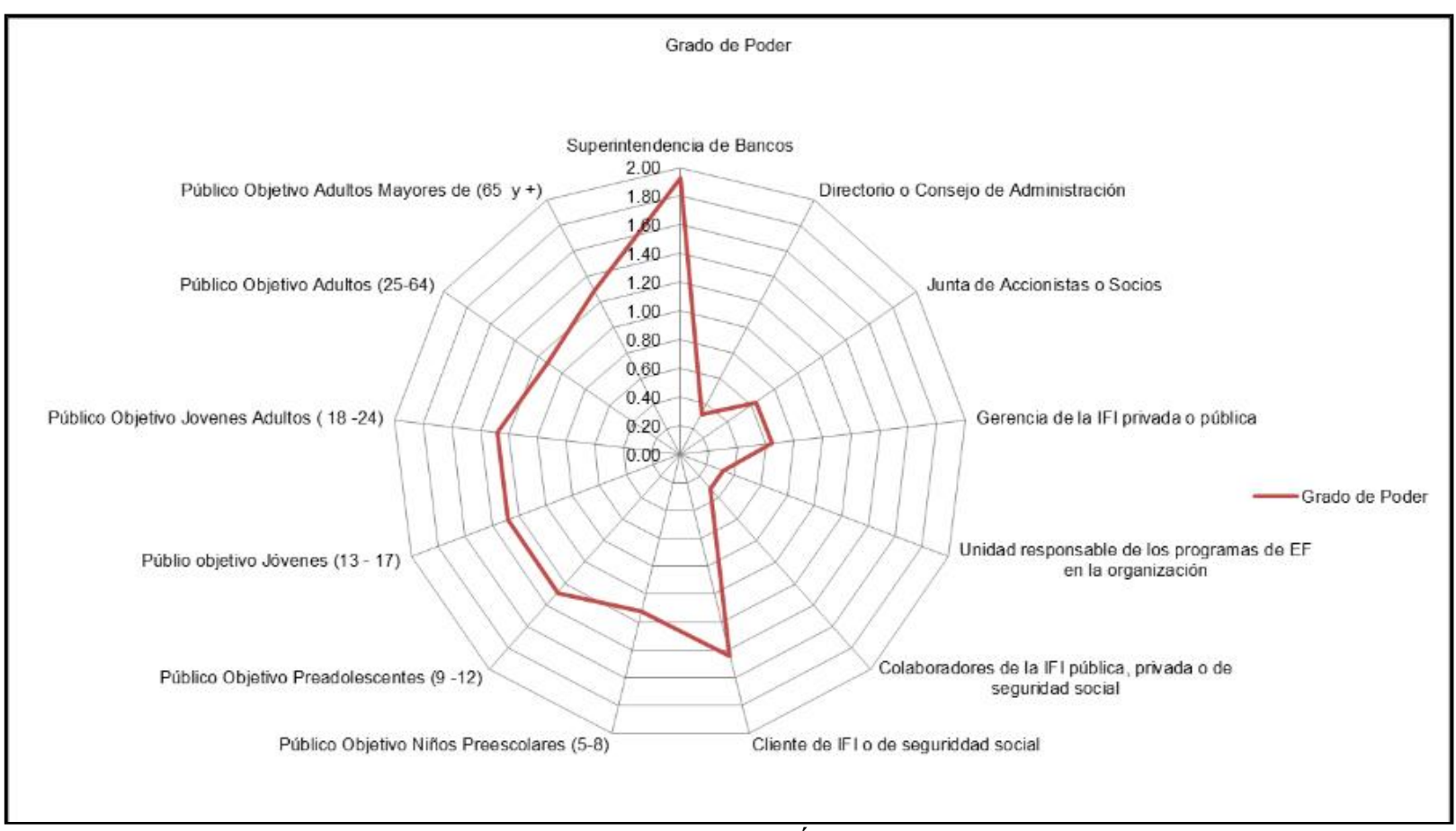

Gráfico $N^{\circ}$ 2. Stakeholders Según El Índice De Preponderancia

Fuente: Elaboración propia

De tal manera, que una vez consolidad la información, se puede evidenciar que los stakeholders de mayor importancia son la Superintendencia de Bancos, y todos y cada uno de los públicos objetivos según sus distintos rangos etarios.

\section{Discusión}

Vale recordar que la educación financiera es un conjunto de conocimientos y habilidades que están presentes de manera permanente en la vida de los individuos, ya sea a través de decisiones respecto de cómo financiar su educación, su vivienda, la seguridad social, planes de salud, etc.

Por tanto no se está hablando únicamente de intereses personales o familiares, si no que al evaluar a los individuos en su conjunto se está hablando de la creación una ciudadanía con amplias oportunidades de participación de manera informada y responsable respecto de sus opciones financieras.

Es así que tomando en consideración que los resultados de este estudio manifiestan que los stakeholders de mayor importancia son la Superintendencia de Bancos, y cada uno de los públicos objetivos según sus distintos rangos etarios, es necesario designar de manera formal a esta Superintendencia como quien lidera la formulación, implementación y evaluación de la política de educación financiera. De esta manera podrá no solamente supervisar a las instituciones que están actualmente bajo su control, sino que también podrá exhortar a otras entidades a que sus actividades contemplen la difusión de los fundamentos de la educación financiera. 
Contar con la Superintendencia de Bancos como principal stakeholder realmente amplía los alcances de la responsabilidad social entre entidades que están bajo su supervisión como son Bancos, Sociedades Financieras, Mutualistas, Emisores de Tarjetas de Crédito, Burós de Información Crediticia, Instituciones Financieras Públicas como la Corporación Financiera Nacional, BanEcuador, Banco del Estado, Banco Central, Institutos de Seguridad Social como ISSPOL, ISSFA, IESS, etc. (Superintendencia de Bancos, 2009). Ya que además de dar cumplimiento a los requisitos que dispone la autoridad reguladora, se estará satisfaciendo a clientes y a la comunidad en general con la transferencia de conocimientos y habilidades que orienten importantes decisiones asociadas a la vida económica de los individuos.

Adicionalmente, otro grupo de stakeholders muy importante es el representado por los distintos grupos etarios. Con lo que se demuestra que la importancia de la educación financiera se extiende a lo largo de la vida de las personas. Por lo que para la difusión de los programas de educación financiera se requiere trabajar de manera conjunta con instituciones que puedan hacer llegar estos contenidos al mayor número de individuos, sea a través de las instituciones educativas, aseguradoras, cooperativas de ahorro y crédito, ministerios, etc. y así asegurarse que los contenidos puedan llegar a ser interiorizados por los distintos públicos objetivos.

\section{Conclusiones}

De acuerdo a la información examinada y los resultados que son producto de la identificación de los stakeholders se tiene como conclusiones los siguientes puntos:

1. Entre los distintos documentos normativos revisados, no se evidencia una directriz específica para que los grupos de interés colaboren en torno a un mismo objetivo, lo que no asegura el cumplimiento de los preceptos básicos de educación financiera orientados hacia el Buen Vivir.

Es decir, no se evidencia entre estos documentos la clara designación de liderazgo por parte de una entidad a la que se delegue como responsable de la formulación, implementación y evaluación de la política de educación financiera.

2. A través del presente estudio se determina que los principales stakeholders son la Superintendencia de Bancos, y todos y cada uno de los públicos objetivos según sus distintos rangos etarios.

Ante este hallazgo se vuelve necesario empoderar a la Superintendencia de Bancos como responsable de la formulación, implementación y evaluación de la política de educación financiera. De esa manera, ya no sólo se encargará de evaluar el grado de cumplimiento de los programas de educación financiera vigentes entre las entidades sobre las que tiene control. Si no que también será el encargado de establecer vínculos con otras instituciones que puedan convertirse en brazos ejecutores de la educación financiera entre los distintos grupos etarios.

Teniendo como primeros agentes a las entidades financieras bajo su supervisión, que además de cumplir con los requerimientos de la autoridad reguladora, perciben el incentivo de ser responsables con la comunidad en la que interactúan. 
3. La Superintendencia de Bancos está llamado a coordinar la formulación, implementación y evaluación de la política de educación financiera en el país.

Considerando que la educación financiera tiene un amplio público objetivo, debe convertirse en un eje transversal dentro del sistema educativo ecuatoriano con la finalidad de reducir las barreras de acceso a programas y contenidos educativos que difundan y fortalezcan la educación financiera. Por lo que también se necesita que al momento que la Superintendencia de Bancos asume como responsable de esta política, pueda coordinar con el Ministerio de Educación y el Consejo de Educación Superior la inclusión de la educación financiera como parte del programa de estudios en el sistema educativo de escuelas y colegios, además del sistema universitario.

\section{Bibliografía}

Coates, K. (2009). Educación Financiera: Temas y Desafios para América Latina. Conferencia Internacional OCDE- Brasil sobre Educación Financiera. [Diapositivas de PowerPoint].Organisation for Economic Co-Operation and Development. Conferencia llevada a cabo en Río de Janeiro, Brasil. Recuperado de http://www.oecd.org/finance/financialeducation/44264471.pdf

Congreso Nacional. (2000). Ley Orgánica de Defensa del Consumidor. Publicado en Registro Oficial Suplemento No. 116 del 10 de julio de 2000. Última modificación 2011. Recuperado de Http://Www.Industrias.Gob.Ec/Wp-Content/Uploads/2015/04/A2-Ley-Organica-DeDefensa-Del-Consumidor.Pdf

Falçao Martins, H. \& Fontes Filho, J. (1999). ¿En quién se pone el foco? Identificando "stakeholders" para la formulación de la misión organizacional. Revista del CLAD Reforma y Democracia, No. 15 (Oct. 1999). Caracas. Recuperado de http://siare.clad.org/revistas/0033942.pdf

García, N. Grifoni, A. \& López, J. (2013). La educación financiera en América Latina y el Caribe. Corporación Andina de Fomento. Recuperado de https://www.oecd.org/daf/fin/financialeducation/OECD_CAF_Financial_Education_Latin_AmericaES.pdf

Junta Bancaria. (2013). Resolución No. JB-2013-2393 del 22 de enero de 2013. Recuperado de http://www.sbs.gob.ec/medios/Portaldocs/downloads/normativa/nueva_codificacion/todo s/L1_XIV_cap_IV.pdf

Mitchell, R. Agle, B. and Wood, D. (1997). Toward a Theory of Stakeholder Identification and Salience: Defining the Principle of Who and What Really Counts. The Academy of Management Review, Vol 22, No. 4 (Oct. 1997). pp. 853-886. Recuperado de http://courses.washington.edu/ilis580/readings/Mitchell_et_al_1997.pdf

Secretaría Nacional de Planificación y Desarrollo - Senplades. (2013). Plan Nacional de Desarrollo / Plan Nacional para el Buen Vivir 2013-2017. Recuperado de 
http://documentos.senplades.gob.ec/Plan\%20Nacional\%20Buen\%20Vivir\%2020132017.pdf

Superintendencia de Bancos y Seguros (2015). Resolución No. SB-2015-665 del 17 de agosto de 2015. Recuperado de http://www.bde.fin.ec/sites/default/files/Resolucio\%CC\%81n\%20SB-2015665\%20de\%2017\%20de\%20agsoto\%20de\%202015.pdf

Superintendencia de Bancos del Ecuador. (2009). Preguntas Frecuentes. Generalidades del Sistema Financiero - ¿Quiénes integran el Sistema Financiero Ecuatoriano? Recuperado de

http://www.superbancos.gob.ec/practg/sbs_index?vp_art_id=1\&vp_tip=11\&vp_lang=1\& vp_buscr=11\#131

Weimer, D. \& Vining, A. (1989). Policy Analysis. Concepts and practices. New Jersey: Prentice Hall. 